Provided for non-commercial research and educational use only. Not for reproduction or distribution or commercial use.

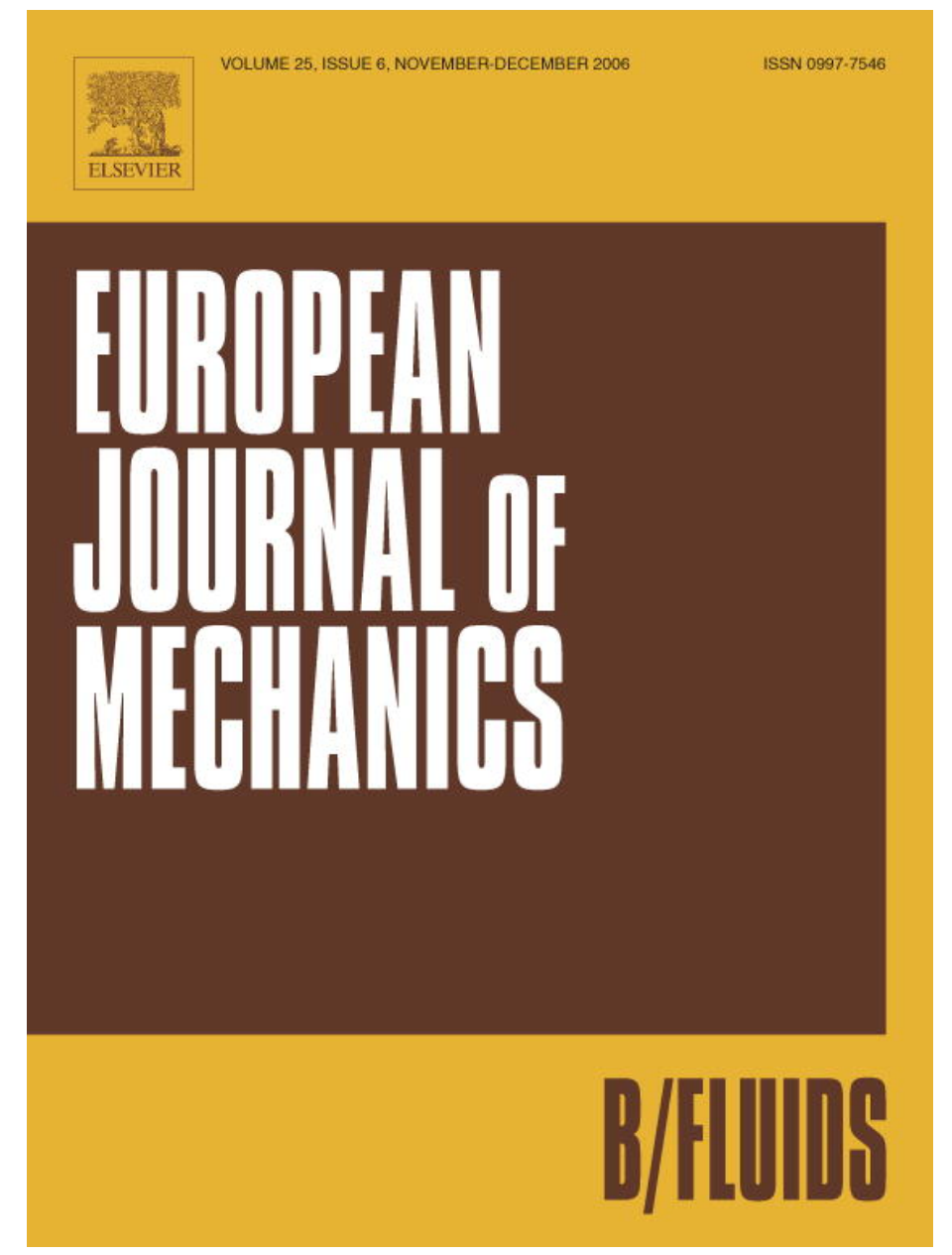

This article was originally published in a journal published by Elsevier, and the attached copy is provided by Elsevier for the author's benefit and for the benefit of the author's institution, for non-commercial research and educational use including without limitation use in instruction at your institution, sending it to specific colleagues that you know, and providing a copy to your institution's administrator.

All other uses, reproduction and distribution, including without limitation commercial reprints, selling or licensing copies or access,

or posting on open internet sites, your personal or institution's website or repository, are prohibited. For exceptions, permission may be sought for such use through Elsevier's permissions site at: 


\title{
Underbody and ground effects on rotating disc flow: a global scale inviscid study
}

\author{
R. Purvis ${ }^{\mathrm{a}, *}$, E.R. Johnson ${ }^{\mathrm{b}}$, F.T. Smith ${ }^{\mathrm{b}}$ \\ ${ }^{a}$ School of Mathematics, University of East Anglia, Norwich, NR4 7TJ, UK \\ ${ }^{\mathrm{b}}$ Department of Mathematics, University College London, Gower Street, London, WC1E 6BT, UK
}

Received 9 December 2003; received in revised form 11 July 2005; accepted 29 August 2005

Available online 21 June 2006

\begin{abstract}
The flow induced by a finite disc rotating near horizontal ground is considered, including the effects of an underbody. This paper concentrates on determining the shape of the free layer beyond the rim of the disc which is horizontal in the absence of the underbody and ground but forced to deform to ensure that conditions across the layer are satisfied when the underbody or ground is added. The far-field behaviour, the inviscid flow produced by a nominally infinite disc near the ground and the global solution for small ground clearances are considered analytically, and the full problem is posed as an integral problem. This is then solved numerically and analytically. Results are presented for various heights of the disc above the ground and for discs with an axisymmetric underbody present. A universal form is found for the farfield shape (which is controlled by entrainment into the free layer) but both the underbody and the ground effects are found to increase very significantly for reduced clearances.
\end{abstract}

(c) 2006 Elsevier Masson SAS. All rights reserved.

Keywords: Rotating disc; Ground effects; Inviscid; Underbody

\section{Introduction}

The flow produced by a finite rotating disc immersed in fluid, with or without an underbody, under the influence of horizontal ground beneath, is the present concern. As well as intrinsic interest in the problem and subsequent methodology there is practical interest also, mostly in terms of rotor flows. For numerous rotor blade and similar motions flight characteristics during hovering or near-hovering conditions are significant for take off and landing close to the ground or a floating platform. See for example Bramwell [1], Seddon [2], Newman [3] and Conlisk [4].

Global features. In the many interesting computational as well as other studies of rotor blade flows a common approach is to concentrate on the complex local motions possible near a helicopter blade for instance, such as with tip vortices and blade-vortex interaction. Our approach is to start more simply in order to increase understanding of the total flow structure, especially given that the typical Reynolds number $R e$ is usually medium to large in the practical context. Thus the model adopted here is aimed at shedding light on global effects. The direct application to helicopter blade flows may be limited but a global picture of the flow in this context can then emerge.

\footnotetext{
* Corresponding author.

E-mail address: r.purvis@uea.ac.uk (R. Purvis).
} 
On motivation and issues. The current theoretical research, then, is on the modelling of basic effects due to an underbody and to the presence of ground effect on the air flow induced by a horizontal disc with vertical axis of symmetry. As hinted already, the flow in reality is complex, three-dimensional, unsteady and probably turbulent for the most part. On the other hand, comparatively little research of a theoretical nature appears to have been done on the fundamental flow mechanics involved in the present body-to-ground interactions on a global scale, although contributions on various related more local aspects including internal flow interactions have been made by Zbrozek [5], Widnall and Barrows [6], Lighthill [7], Newman [8], Tuck and Bentwich [9], Jones [10] and Jones and Smith [11]. Accordingly, it seems sensible to tackle first the inviscid, incompressible range of steady laminar motions, in our case with axisymmetry.

To emphasise, the major issues concern the following: the precise farfield behaviour; the overall influence of an underbody beneath the disc; the effects produced at the ground by the presence of the body, and vice-versa; exactly how those effects change with closeness to the ground; and the corresponding global flowfield responses. Results or improved models regarding these issues will provide first steps towards better understanding of the complete fluid dynamics inherent in the complex motions that are encountered in practice, answering questions such as how well an inviscid model captures the main physics involved.

Disc-ground interaction. As a first step towards understanding the ground effect on a general rotating blade system, the finite horizontal circular disc is taken to be rotating with a uniform angular velocity at a constant distance above horizontal ground. This is of interest not just because it has a broad similarity to the general rotor system but also as it has some more direct connections. Specifically, the far field response of any slender bounded rotor system is the same as for the disc (Smith and Timoshin [12]) since any azimuthal dependence erodes away with increasing distance from the rotor axis. So the responses here are relevant to a system with genuine blades and wakes present, c.f. the actuator disc approximation (Glauert [13]). Another limit of particular relevance is that of a rotor with many blades. In this case (again see Smith and Timoshin [12]) the flow is found to have a two-tiered structure consisting of a fast varying inner tier incorporating the local blade-wake interactions and a slowly varying outer tier containing mean Von Karman flow, where the viscous Von Karman flow is the well-known solution for an infinite rotating disc in free space. Therefore from outside the boundary layer we effectively see the same solution in both the many-blade and the present configurations.

For a finite disc rotating with uniform velocity in free space the flow response is well known. The disc acts as a centrifugal pump, pulling fluid vertically into the disc boundary layer and throwing it out radially in a thin entraining layer. For a disc near the ground the viscous boundary layer part of the calculation is the same as in the free case; the viscous layer on the disc still pulls in the same amount of fluid and creates a thin layer beyond its rim. However, consideration of the outer inviscid flow clearly shows that, due to the influence of the ground, the free layer shape can no longer remain flat without violating the required pressure and entrainment conditions across the wake.

Our aim therefore is to investigate what influence the presence of the ground has on the shape of the free layer/wake produced by a finite rotating disc with or without an underbody.

The radial and vertical velocities are given by $U(r, z)$ and $V(r, z)$ respectively, and $R e$ is the non-dimensional Reynolds number based on $\Omega$, the angular velocity, $R$, the disc radius, and $v$ the kinematic viscosity of the fluid. We assume $R e=\Omega R^{2} / v \gg 1$.

The non-dimensionalised flow geometry (see Fig. 1) is that of an impermeable disc of radius unity rotating at an $\mathrm{O}(1)$ height $h$ above the ground. This generates a thin entraining viscous layer on the disc and continuing beyond the disc rim. The unknown shape of this curve is described as $C$. The entrainment velocity into the lower side of the layer is denoted by $R e^{-1 / 2} v_{e}^{-}(s)$ and into the upper side is $R e^{-1 / 2} v_{e}^{+}(s)$ where $s$ is the distance along $C$ from the axis of the disc. The unit normal $\hat{\mathbf{n}}$ to the curve and disc is chosen so that $\hat{\mathbf{n}} \cdot \hat{\mathbf{z}}>0$ where $\hat{\mathbf{z}}$ is a unit vector along the $z$-axis. The disc can itself be attached to an arbitrary stationary axisymmetric body (modelling the body under a rotor system), which of course affects the whole motion, a case which is taken up later in the paper; the combined boundary of the body and the disc is then denoted by $H$ and $\hat{\mathbf{n}}$ describes the normal to the layer, disc and body.

The ratio $H / R$. The assumed thin viscous layers on the underbody and on the ground are neglected here; in practice there can be significant boundary layers on both. The regime of interest here is for moderate values of $h / R$. For small values $\left(\mathrm{O}\left(R e^{-1 / 2}\right)\right.$ or less $)$ of this ratio the entire flow field is rotational rather than having distinct $\mathrm{O}\left(R e^{-1 / 2}\right)$ boundary layers being formed on the ground and the disc separated by an irrotational core. In this situation our assumption of an irrotational core becomes invalid as the entire core will be rotational. The flow might be partly rotational even for ground clearances larger than the above boundary layer thickness, depending on the large-distance conditions such 


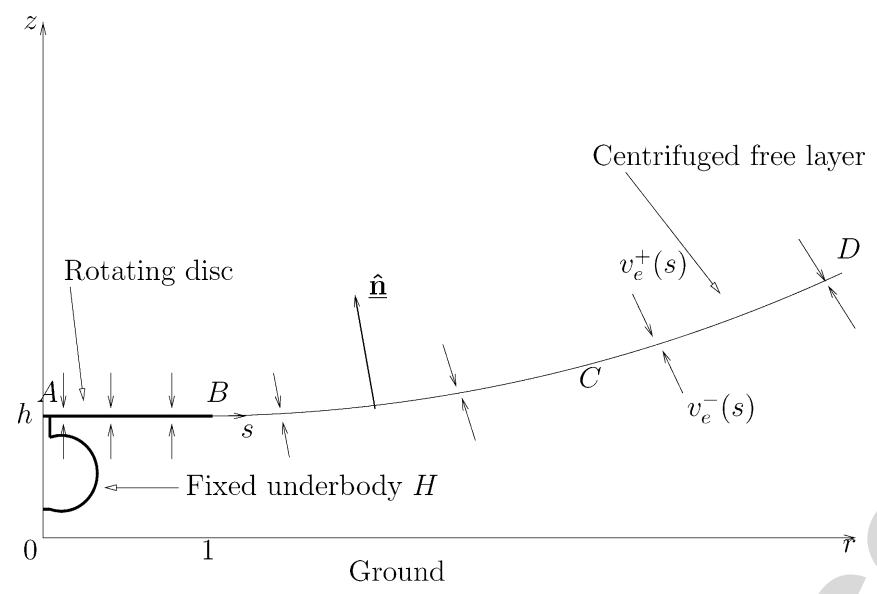

Fig. 1. The flow geometry: a finite horizontal disc rotating at a constant distance $h$ above horizontal ground, together with an arbitrary fixed body shape $H$.

as in cases of radial inflow or wakes as well as the possible occurrence of separation or the presence of turbulent flow for instance. The order of magnitude for the extent of any such rotational parts of the flow field has not yet been determined by theory. Overall the relevance of the present model clearly requires the ratio $h / R$ to be sufficiently high. Indeed, Owen and Rogers [14] demonstrate that for a small $h / R$ ratio $(=0.1)$ the central core rotates as a solid body with constant, rather than negligible, vorticity. As $h / R$ becomes large the symmetric case is recovered with the flows on the two sides of the disc becoming similar. Altogether the current approach assumes that $h / R$ is large enough with respect to $R e^{-1 / 2}$ or other relevant boundary layer thickness that the core remains inviscid but small enough that the flow field beneath the disc is significantly changed.

Important role of entrainment velocity. Including entrainment requirements is essential because they affect the global motion significantly in the far field, in line with the present emphasis on modelling the global features, although there are in addition pressure requirements. The latter tend to be more substantial closer to the rim of the disc, as in the Smith [15] study of the trailing edge interaction there, and so here a composite approach is eventually adopted to include both requirements. The approach is likely to model well the realistic case of turbulent layers also.

In Section 2 below we set out the problem and examine two limits of interest: namely that of the large $r$ response of the layer shape and the flow induced by a nominally infinite disc near the ground. Given this starting point we then turn, in Section 3, to the full problem of determining the complete layer shape from the disc rim to the far field. An integral equation is derived which leads to a minimisation problem being posed to calculate the required shape. Results are presented for various $h$ values, first for the disc with no body shape present and secondly for the case with a body shape beneath to investigate what influence this has on the shape of the viscous shear layer. The influence of the magnitude of the pressure jump across the layer is also examined. The case of small $h$ is then considered in Section 3.3 and the layer shape is shown to have infinite curvature at the disc rim, a property which compares well with the results from the integral equation calculations. Finally, further comments are presented in Section 4.

\section{The problem}

\subsection{Governing equations}

As the inviscid flow of interest here is driven at first by matching with the entrainment velocities into the boundary layer we expand the velocities and pressure as

$$
[U, V, P](r, z)=\left[0,0, p_{\infty}\right]+\left[\epsilon u, \epsilon v, \epsilon^{2} p\right]+\cdots,
$$

where $\epsilon=R e^{-1 / 2}$. Substituting these into the axisymmetric Navier-Stokes equations along with the assumption that $R e \gg 1$ leaves us with the axisymmetric Euler equations for $u, v$ and $p$ :

$$
\frac{\partial u}{\partial r}+\frac{u}{r}+\frac{\partial v}{\partial z}=0
$$




$$
\begin{aligned}
& u \frac{\partial u}{\partial r}+v \frac{\partial u}{\partial z}=-\frac{\partial p}{\partial r} \\
& u \frac{\partial v}{\partial r}+v \frac{\partial v}{\partial z}=-\frac{\partial p}{\partial z}
\end{aligned}
$$

We assume initially that the flow is irrotational and by the principle of conservation of vorticity it will then remain so for all time. This is thought to be a physically valid assumption except for when the aspect ratio becomes small when rotational effects will dominate the entire gap between disc and wall; we refer here also to the comments in Section 1. It is therefore possible to pose the full problem in terms of the velocity potential $\Phi$ where $u=\partial \Phi / \partial r$ and $v=\partial \Phi / \partial z$. The velocity potential, continuous through all space outside of the body $H$, satisfies

$$
\nabla^{2} \Phi=\frac{\partial^{2} \Phi}{\partial r}+\frac{1}{r} \frac{\partial \Phi}{\partial r}+\frac{\partial^{2} \Phi}{\partial z^{2}}=0 \quad \text { in } r \geqslant 0, z \geqslant 0
$$

with

$$
\begin{aligned}
& \frac{\partial \Phi}{\partial z}=0 \quad \text { on } z=0, r \geqslant 0, \\
& \frac{\partial \Phi}{\partial n}=0 \quad \text { on } H, \\
& \frac{\partial \Phi}{\partial n}=-v_{e}^{+}(s) \quad \text { on } A B D^{+}, \\
& \frac{\partial \Phi}{\partial n}=v_{e}^{-}(s) \quad \text { on } A B D^{-} .
\end{aligned}
$$

These conditions are respectively the inviscid tangential flow condition on the ground, on the body, and the entrainment into the boundary layer along either side of the disc and the free layer or wake. In the full problem there may be some inner-outer interaction, causing the entrainment velocities $v_{e}^{ \pm}$to depend upon the layer shape $Z(r)$ but we are going to model the entrainment velocities as known and the boundary layer flow to be independent of the shape of the viscous shear layer. We also have a relation on the pressures at the wake. The required pressure jump across the layer is modelled by

$$
p^{+}-p^{-}=[p]=-A(r) \frac{Z^{\prime \prime}(r)}{\left(1+\left(Z^{\prime}(r)\right)^{2}\right)^{3 / 2}},
$$

where the function $A(r)$ is determined from the viscous shear layer. This model is based upon Papageorgiou and Smith [16] and is an attempt to introduce a pressure jump into the problem. Roughly $A(r) \approx \bar{u}^{2} \delta$ where $\bar{u}$ is the radial velocity in the viscous layer and $\delta$ is the viscous layer thickness. Typically $\bar{u}$ decays as $1 / r$ while $\delta$ grows as $r$, in the laminar regime. Hence we assume $A(r)=\alpha / r$ (cf. (11) below) where $\alpha$ is a constant, assumed known from the boundary layer flow. We will consider solutions for a variety of magnitudes of $\alpha$.

For a rotating disc in the absence of ground effect the entrainment velocities are given by

$$
v_{e}^{+}=v_{e}^{-}= \begin{cases}\gamma & \text { on } A B \\ \gamma / r & \text { on } C\end{cases}
$$

where $\gamma$ is a constant. The first relation in (11) comes from the Von Karman solution and the second is from Smith and Timoshin [12] who show that the decay in entrainment of fluid into the viscous layer varies essentially as $1 / r$. In more detail, the entrainment velocities vary smoothly, with a small region over the disc rim smoothly connecting the entrainment from the constant velocities along the disc to the inverse-distance decay in the layer beyond the rim. As this region is very local it is taken not to have an impact on the global solution. Again the choice of the positive constant $\gamma$ is unimportant in the current model and is, for convenience, set as $\gamma=1 / 2$.

\subsection{The far field response}

From the far field the disc and the ground can be viewed as effectively coincident, leaving two distinct regions: one above and one below the entrainment layer which is assumed to asymptote to a straight line through the origin of an unknown slope (given by an angle $\alpha$ ). The far-field problem to be solved, in terms of the velocity potential $\Phi$, but in spherical coordinates $(s, \theta)$, is given by (5) subject to 


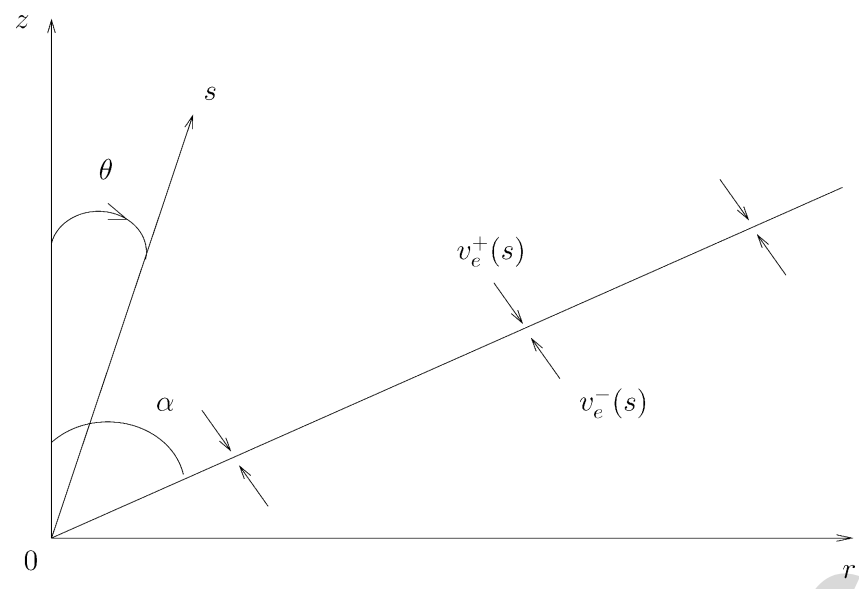

Fig. 2. The far-field problem.

$$
\begin{aligned}
& \frac{\partial \Phi}{\partial \theta}=0 \quad \text { when } \theta=0, \\
& \frac{\partial \Phi}{\partial \theta}=0 \quad \text { when } \theta=\frac{\pi}{2}, \\
& \frac{\partial \Phi}{\partial \theta}= \pm \gamma \quad \text { when } \theta=\alpha^{\mp},
\end{aligned}
$$

which are a symmetry condition, the no-penetration ground condition and the requirement of entrainment velocities into the layer, respectively (see Fig. 2). The angle $\alpha$ is the inclination of the layer to the vertical and is to be determined. We also require the pressure to be continuous across the layer as the curvature is zero here. Solving these equations yields the velocity potential $\Phi$ as

$$
\Phi= \begin{cases}-A_{1}\left[\ln (s \sin \theta)-\frac{1}{2} \ln \left(\frac{1-\cos \theta}{1+\cos \theta}\right)\right] & \text { if } \theta<\alpha \\ -A_{2} \ln (s \sin \theta) & \text { if } \theta>\alpha\end{cases}
$$

and Stokes's streamfunction $\Psi$ as

$$
\Psi= \begin{cases}A_{1} s[\cos \theta-1] & \text { if } \theta<\alpha, \\ A_{2} s \cos \theta & \text { if } \theta>\alpha\end{cases}
$$

respectively, where $A_{1}, A_{2}$ are constants adjusted to satisfy the entrainment condition (14). The deflection angle $\alpha$ is now determined from applying Bernoulli's theorem along the layer. This gives the requirement that $\left(\frac{\partial \Phi}{\partial s}\right)^{2}+\left(\frac{1}{s} \frac{\partial \Phi}{\partial \theta}\right)^{2}$ needs, in order to give pressure continuity across the layer, to be the same on either side, i.e. at $\theta=\alpha^{ \pm}$. Applying this it becomes apparent that this can only be satisfied for

$$
\alpha=\frac{\pi}{3} .
$$

These solutions form the far-field solution for any bounded rotating blade system in the proximity of the ground. It holds for the rotating disc considered here but, to repeat, it also holds when there are genuine blades and wakes present as in a rotor blade system, because the azimuthal dependence erodes away as $r$ increases, leaving the same far-field form regardless of the initial rotor set-up.

\subsection{Flow induced by an 'infinite' disc rotating above the ground}

We temporarily consider an unbounded rotating disc near the ground, or strictly disc of large radius. This is of interest as it is gives an idea of what is happening beneath a finite disc near to the ground and is useful in the small $h$ analysis near the disc rim considered in Section 3.3. Above the disc we have the Von Karman solution with fluid constantly flowing in axially. However, beneath the disc the no penetration condition at the ground prevents this from happening; so what form does the flow take there? For further clarification and to avoid any contradiction, we remark that the disc radius while large compared with the ground clearance is supposed to be much less than that at which viscous and other rotational effects can enter play. This is in line with comments in Section 1. 
We have to solve the Euler equations in the region between the disc and the ground subject to no penetration on the ground and constant entrainment into the layer on the disc. We consider $r \gg 1$ and, assuming $v$ is independent of $r$ at large distances, we write

$$
v(r, z)=\bar{v}(z),
$$

which, along with the Euler equations, imply the forms

$$
\begin{aligned}
& u(r, z)=r \bar{u}(z), \\
& p(r, z)=r^{2} \bar{p}+p_{0}(z),
\end{aligned}
$$

where $\bar{p}$ is a constant. Substituting these into the Euler equations reduces them to

$$
\begin{aligned}
& 2 \bar{u}+\bar{v}^{\prime}=0, \\
& \bar{u}^{2}+v \bar{u}^{\prime}=-2 \bar{p}, \\
& \bar{v} \bar{v}^{\prime}=-p_{0}^{\prime},
\end{aligned}
$$

subject to $\bar{v}(h)=\gamma, \bar{v}(0)=0$, where $\gamma$ is the constant entrainment into the discs boundary layer and ' denotes differentiation with respect to $z$. Integrating (22) immediately yields $p_{0}=-\frac{1}{2} \bar{v}^{2}$. Differentiating (21), substituting for $\bar{v}^{\prime}$ from (20) and solving the resulting differential equation yields the simple form for the velocities and pressure as

$$
u=-\frac{\gamma r}{2 h}, \quad v=\frac{\gamma z}{h}, \quad p=-\frac{r^{2} \gamma^{2}}{8 h^{2}}-\frac{\gamma^{2} z^{2}}{2 h^{2}},
$$

assuming a zero vorticity condition $u^{\prime}=0$. So this determines the flow between an infinite disc and the ground, or the flow beneath a disc close to the ground but away from the disc rim. See also Debuchy et al. [17] who consider a similar limit for two infinite co-rotating discs at small $h$.

\section{Determining the layer shape}

\subsection{An integral equation for the layer shape}

We now seek to derive an integral formulation of the global problem in order to find the layer shape between the disc rim and the far-field form determined above. Let $G\left(r, r_{0}, z, z_{0}\right)$ be the Green's function satisfying

$$
\nabla^{2} G=\delta\left(z-z_{0}\right) \delta\left(r-r_{0}\right) / r_{0},
$$

so that $G$ is the velocity potential at $(r, z)$ due to a ring source of total strength $2 \pi$ at $\left(r_{0}, z_{0}\right)$. Two forms for $G$ are

$$
G\left(r, r_{0}, z, z_{0}\right)=\frac{1}{4} \int_{0}^{\infty} J_{0}\left(k r_{0}\right) J_{0}(k r) \mathrm{e}^{\left(-k\left|z-z_{0}\right|\right)} \mathrm{d} k=\frac{2}{\pi R_{1}} K\left(\frac{2 \sqrt{r r_{0}}}{R_{1}}\right),
$$

where $K$ is the complete elliptic integral of the first kind and $R_{1}^{2}=\left(r+r_{0}\right)^{2}+\left(z-z_{0}\right)^{2}$. The first of these is from Morse and Fechbach [18] who derive it from the streamfunction for a ring source and the second is from an integral relation given in Gradstein and Ryzhik [19]. The Green's function thus has a single logarithmic singularity when simultaneously $r=r_{0} \neq 0$ and $z=z_{0}$. The second form listed in (25) is the most useful for our purposes. Let $C$ be described as $z=Z(r)$.

Now consider the source velocity potential

$$
\begin{aligned}
\Phi_{S}(r, z)= & -\int_{C} r_{0}\left[v_{e}^{-}\left(s_{0}\right)+v_{e}^{+}\left(s_{0}\right)\right] G\left(r, r_{0}, z, Z\left(r_{0}\right)\right) \mathrm{d} s_{0} \\
& -\int_{C} r_{0}\left[v_{e}^{-}\left(s_{0}\right)+v_{e}^{+}\left(s_{0}\right)\right] G\left(r, r_{0}, z,-Z\left(r_{0}\right)\right) \mathrm{d} s_{0} .
\end{aligned}
$$

The first integral gives a distribution of ring sources of total strength $-2 \pi r_{0}\left(v_{e}^{-}+v_{e}^{+}\right)$per unit distance along $C$ and the second the image of these ring sources in the plane $z=0$. This form for $\Phi_{S}$ satisfies the governing equation, the 
ground condition and gives pressure continuity across the layer. The jump in $\partial \Phi_{S} / \partial n$ in crossing from $z>Z(r)$ to $z<Z(r)$ is now

$$
\left[\frac{\partial \Phi_{S}}{\partial n}(s)\right]_{C}=-\left[v_{e}^{-}(s)+v_{e}^{+}(s)\right]
$$

with $\Phi_{S}$ and its derivatives continuous away from $C$.

In order to allow for a pressure jump across the layer as in (10) we also introduce a sheet of ring vortices along the layer. This, given the correct strength of vortices, keeps the normal velocity into the layer unchanged while introducing a jump in the tangential velocity and therefore $p$. So we also need to determine $\Phi_{v}$, the potential due to this series of ring vortices with unknown strength to give the necessary jump in $u$. In order to achieve this we introduce

$$
\underline{r}_{ \pm}(n)=(r, z) \pm \underline{\hat{n}} \eta,
$$

so that $\underline{r}_{+}, \underline{r}_{-}$are displaced normally off the sheet by a small distance $\eta$, where $(r, z)$ is the point at which we are trying to find $\partial \phi_{v} / \partial n$. We then have

$$
\begin{aligned}
\left(\frac{\partial \Phi_{v}}{\partial r}, \frac{\partial \Phi_{v}}{\partial z}\right)= & \frac{1}{2} \lim _{\eta \rightarrow 0_{+}} \int_{1}^{\infty} V^{R}\left(r_{0}\right)\left(\underline{G}_{1}\left(\underline{r}_{+}, r_{0}, Z\left(r_{0}\right)\right)+\underline{G}_{1}\left(\underline{r}_{-}, r_{0}, Z\left(r_{0}\right)\right)\right) \mathrm{d} r_{0} \\
& +\frac{1}{2} \lim _{\eta \rightarrow 0_{+}} \int_{1}^{\infty} V^{R}\left(r_{0}\right)\left(\underline{G}_{1}\left(\underline{r}_{+}, r_{0}, Z\left(-r_{0}\right)\right)+\underline{G}_{1}\left(\underline{r}_{-}, r_{0}, Z\left(-r_{0}\right)\right)\right) \mathrm{d} r_{0},
\end{aligned}
$$

where $V^{R}(r)$ is the strength of the vortex rings required in order to satisfy (10) and can be determined for a given $Z(r)$, the second integral gives the effect of the image system and $\underline{G}_{1}$ is discussed below. The streamfunction for a single vortex ring is given by Lamb [20] as

$$
\psi\left(r, z, r_{0}, z_{0}\right)=\left(r r_{0}\right)^{1 / 2}\left\{\left(\frac{2}{k}-k\right) K(k)-\frac{2}{k} E(k)\right\},
$$

where

$$
k=\frac{\left(r-r_{0}\right)^{2}+\left(z-z_{0}\right)^{2}}{\left(r+r_{0}\right)^{2}+\left(z-z_{0}\right)^{2}}
$$

and $K(k)$ and $E(k)$ are the complete elliptic functions of the first and second kind respectively. Using this, $\underline{G}_{1}$ can be constructed as

$$
\underline{G}_{1}\left(r, z, r_{0}, z_{0}\right)=\left(\frac{\partial \psi}{\partial z},-\frac{\partial \psi}{\partial r}\right)\left(r, z, r_{0}, z_{0}\right) .
$$

We now have everything we need to calculate $\partial \Phi_{v} / \partial n$, where

$$
\frac{\partial \Phi_{v}}{\partial n}=\frac{\partial \Phi_{v}}{\partial z}-Z^{\prime}(r) \frac{\partial \Phi_{v}}{\partial r},
$$

if we can calculate the integrals on the right-hand side of (29).

This holds for the layer but as yet we have taken no account of the disc (and possible body underneath). Let the normal velocity induced on $H$ by the presence of $\Phi_{S}$ and $\Phi_{v}$ be

$$
u_{n}^{S}(\mu)=\left.\frac{\partial \Phi_{S}}{\partial n}\right|_{H}+\left.\frac{\partial \Phi_{v}}{\partial n}\right|_{H},
$$

where $\mu$ is some convenient coordinate describing the surface $H$. Now define the body velocity potential $\Phi_{H}$ as the half-space potential that, when added to the source-induced flow on $H$, gives the required entrainment velocities there, i.e. satisfying

$$
\begin{aligned}
& \nabla^{2} \Phi_{H}=0, \\
& \frac{\partial \Phi_{H}}{\partial z}=0 \quad \text { on } z=0, r \geqslant 0, \\
& \frac{\partial \Phi_{H}}{\partial n}=-u_{n}^{S}(\mu)-v_{e}^{ \pm}(\mu) \quad \text { on } H,
\end{aligned}
$$


where $v_{e}^{ \pm}(v)$ is non-zero only on the disc $A B$. This linear external Neumann problem can be solved straightforwardly by any convenient method.

The total potential

$$
\Phi=\Phi_{H}+\Phi_{S}+\Phi_{v},
$$

then satisfies the governing equation (5), the no penetration conditions (6), (7) and the pressure condition (10). To satisfy the remaining entrainment conditions on the normal velocities at $C$ it is sufficient to add to the jump condition (27) the requirement that the difference of the entrainment velocities on each side of $C$ is $v_{e}^{-}-v_{e}^{+}$, and so fixing the correct entrainment on either side of the layer. Thus we introduce

$$
\begin{aligned}
\Delta(r)= & \frac{1}{2}\left(\left.\frac{\partial \Phi}{\partial n}\right|_{C^{-}}+\left.\frac{\partial \Phi}{\partial n}\right|_{C^{+}}\right) \\
= & \left.\frac{\partial \Phi_{H}}{\partial n}\right|_{C}-f_{C} r_{0}\left[v_{e}^{-}\left(s_{0}\right)+v_{e}^{+}\left(s_{0}\right)\right] \frac{\partial G}{\partial n}\left(r, r_{0}, z, Z\left(r_{0}\right)\right) \mathrm{d} s_{0} \\
& -\int_{C} r_{0}\left[v_{e}^{-}\left(s_{0}\right)+v_{e}^{+}\left(s_{0}\right)\right] \frac{\partial G}{\partial n}\left(r, r_{0}, z,-Z\left(r_{0}\right)\right) \mathrm{d} s_{0}+\frac{1}{2}\left(\left.\frac{\partial \Phi_{v}}{\partial n}\right|_{C-}+\left.\frac{\partial \Phi_{v}}{\partial n}\right|_{C+}\right),
\end{aligned}
$$

where the first integral is a Cauchy principal value. The necessary conditions are satisfied provided

$$
\Delta(r)=\frac{1}{2}\left(v_{e}^{-}-v_{e}^{+}\right)(r) \quad \text { for all } r \geqslant 1 .
$$

Together (39) and (40) give a singular integral equation determining $Z(r)$ and thus the shape of the layer crosssection $C$.

\subsection{The numerical solution of the integral equation}

The solution of the integral equation (39), coupled with (40), for $\Delta(r)$ is now a numerical task. Before being able to determine $\Delta(r)$ we need to describe $Z(r)$ through some form of parameterisation. We also need an efficient method of obtaining $\Phi_{H}$ and we need to evaluate the integrals on the right-hand side of (29). There are many possible ways of doing these but the methods presented here are thought to be sensible and appear to work well.

As $C$ is expected to be very smooth it seems most efficient to use a Tchebyshev expansion to describe $Z(r)$ since the coefficients of the higher terms will decay exponentially fast, leading to an efficient representation of the layer shape. Further, it is convenient to map the region $1 \leqslant r<\infty$ onto a finite region. To increase the resolution at the edge $B$ (see Fig. 1) where $Z^{\prime}(r)$ changes most rapidly it is convenient to map this point to -1 . Also to avoid unnecessarily high resolution at infinity where $Z^{\prime}(r)$ changes most slowly it is convenient to map this to 0 . The mapping used depends on the value of $h$. For most $h$ values the mapping

$$
\xi=-1 / r \quad(-1 \leqslant \xi \leqslant 0)
$$

is used. However, as will be seen, for $h \ll 1$ it is more useful to use the mapping

$$
\xi=-1 / r^{2} \quad(-1 \leqslant \xi \leqslant 0) .
$$

Whichever is used, we then describe $Z^{\prime}(r)$, the fundamental quantity in (39), as

$$
Z^{\prime}(r)=f(\xi)=\sum_{n=0}^{\infty} a_{n} T_{n}(\xi),
$$

where $T_{n}$ is the $n$th Tchebyshev polynomial

$$
T_{n}(\xi)=\cos \left(n \cos ^{-1} \xi\right) .
$$

Since $f(\xi)$ is a polynomial, $Z(r)$ can be obtained readily from $Z^{\prime}(r)$ by an analytic integration. 
In order to immediately satisfy the far field and disc rim conditions $Z^{\prime}(\infty)=1 / \sqrt{3}$ and $Z^{\prime}(0)=0$, we impose the requirements that $f(-1)=0$ and $f(0)=1 / \sqrt{3}$ and so (43) gives

$$
a_{0}=\frac{1}{\sqrt{3}}-\sum_{n=2}^{\infty} a_{n} T_{n}(0), \quad a_{1}=a_{0}+\sum_{n=2}^{\infty} a_{n} T_{n}(-1) .
$$

The undetermined coefficients are thus $a_{2}, a_{3}, a_{4}, \ldots$, as $a_{0}$ and $a_{1}$ are determined from the linear relations above.

The chosen method for determining the body potential $\Phi_{H}$ is an axisymmetric panel method. See Hess and Smith [21] for a detailed discussion of the method and Hess [22] for a more recent review; we will give a brief overview here. The disc, and if present the body, is described as a series of source panels. The profile curve (i.e. at $\theta=0$ say) is approximated by a series of $J$ line segments. These segments, when rotated through $2 \pi$ radians, describe the entire body. Each of these line segments then describes a line of ring sources with constant, but as yet undetermined, strength $\sigma_{i}$, where $i$ is a typical panel. The centre point of each line segment is called the control point.

The potential $\phi_{1}$ at a point $(r, z)$ due to a ring source of unit strength and radius $a$ lying in the plane $z=b$ is given by

$$
\phi_{1}=\frac{4 a K\left(k_{1}\right)}{\left[(r+a)^{2}+(z-b)^{2}\right]^{1 / 2}}, \quad k_{1}^{2}=\frac{4 a r}{(r+a)^{2}+(z-b)^{2}},
$$

and $K$ is the complete elliptic function. The potential, $\phi^{i k}$ induced at a given control point $i$ due to a different line segment $k$ can then be calculated by simply integrating the relevant equation from (46) along the line segment $k$. The segment $i$ also induces a potential at its own control point. This is somewhat less straight forward as the integrals are singular and must be considered as principal values. The problem is addressed in Hess and Smith [21] where the authors develop a series expansion near to the singular point, which effectively carries out the integrals as principal values, and integrates as before away from the singular point. Using this technique to calculate $\phi^{i i}$, we are in a position to determine the potential and velocities induced at each control point due to the presence of the entire representation of the body. The relevant quantities are given by

$$
\phi^{i}=\sum_{k=1}^{J} \sigma_{k} \phi^{i k}
$$

We can now apply this to our problem (35)-(37). Firstly, in order to satisfy (36) we introduce an image of the body in the plane $z=0$ where the original line segment and its image both have the same strength $\sigma_{i}$. Then we need to determine values of $\sigma_{i}$ such that (37) is satisfied at each control point. This is relatively simple as the required velocities (37), evaluated at each control point, combined with (47) give a set of $J$ linear, algebraic equations for the undetermined $\sigma_{i}$. When solved the $\sigma_{i}$ and source panels give a representation of $\phi_{H}$. Calculation of $\partial \Phi_{H} / \partial n$ on the layer $C$ in (39) can be carried out using these calculated panel strengths and (46).

To evaluate the contribution to $\Delta(r)$ in Eq. (39) of $\Phi_{v}$ we need to evaluate the integrals in (29). In order to do so we make use of the integral formula derived in Bernardinis and Moore [23]. They, in a method akin to that of Hess and Smith [21] for the singularity in the panel method, derive a series expansion for $\underline{G}_{1}$ near the singularity before attempting integration. The integration of this local expansion effectively becomes a straight forward principal value integral which can be calculated analytically and then usual numerical integration elsewhere enables the integrals to be approximated. This method is shown by Bernardinis and Moore [23] to be accurate away from the symmetry line $r=0$ but for $r$ close to zero there is a great loss of accuracy. However, this loss should not be a problem in the current regime as we only need to apply the integral formula for $r \geqslant 1$. The strengths of the vortex rings $V^{R}(r)$ are determined for a given layer shape $Z(r)$ by the pressure requirement (10).

Now for any given $Z(r), \Delta(r)$ can be calculated and so we are in a position to set up an iteration to determine the required layer shape. The iteration is posed as a minimisation of the integral

$$
I=\int_{1}^{\infty}\left[\Delta(r)-\frac{1}{2}\left(v_{e}^{-}-v_{e}^{+}\right)(r)\right]^{2} w(r) \mathrm{d} r,
$$

where $w(r)$ is a weighting function, chosen here to be a sum of delta functions $\delta\left(r_{i}\right)$, corresponding to a least squares minimisation over the collocation points $\left\{r_{i}\right\}$. These are distributed in a similar manner to the mapping (41) or (42), with more points near to the disc rim than in the far field. 


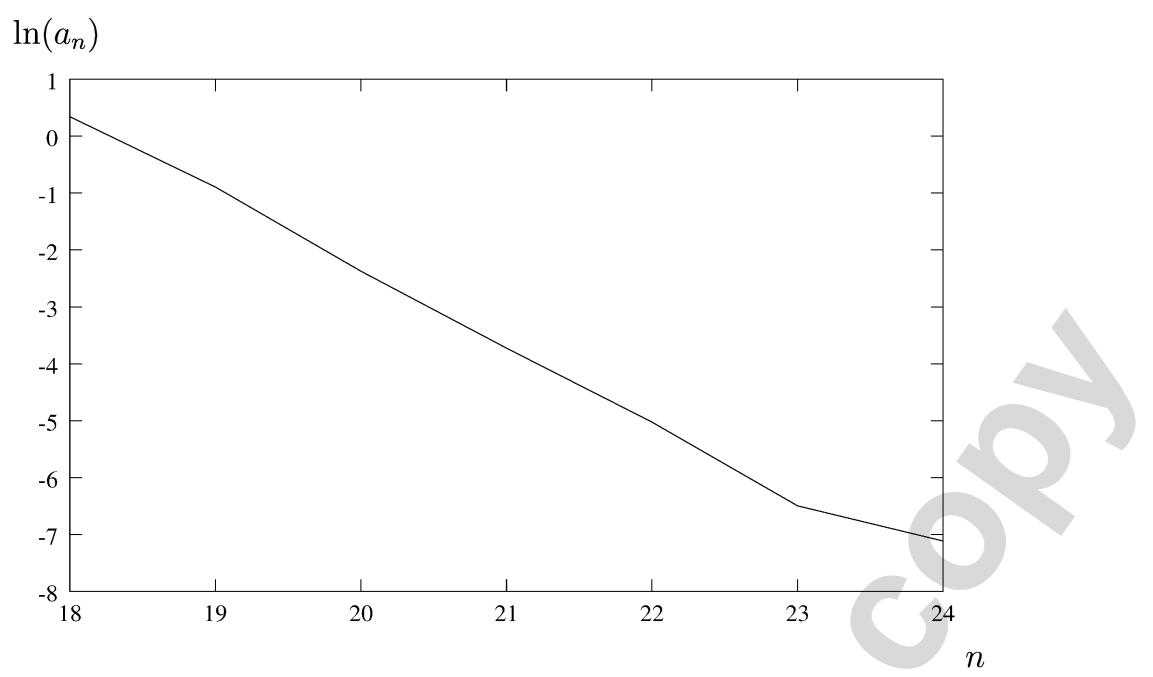

Fig. 3. The expected exponential decay of the Tchebyshev coefficients for the case $h=5$.

For a given set of coefficients, $a_{2}, \ldots, a_{N}$, the value of $I$ can be determined straightforwardly:

(1) First calculate $a_{0}$ and $a_{1}$ from (45) to satisfy the far field and disc rim conditions on the layer shape.

(2) Using the current $Z(r)$ guess, determine the induced velocities at the control points of the panel representation of the body from (37).

(3) With the required control point velocities known, we can solve the necessary linear system to calculate each of the panel strengths $\sigma_{i}$ fixing the body potential $\Phi_{H}$.

(4) It is now possible to calculate $\Delta(r)$ from (39), and hence the value of $I$ can be determined for (48).

(5) Check convergence, then either have a new guess at $a_{2}, \ldots, a_{N}$ and return to item (1) or finish.

The new guess at $a_{2}, \ldots, a_{N}$ required in item (5) is made using the NAG library routine E04FYF which uses a combined Gauss-Newton and modified Newton algorithm. One advantage of the panel method discussed earlier is that the solution of the algebraic equations for $\sigma_{i}$ (item (3) above) is in effect only done once throughout the entire minimisation. The only change at each iteration is the required velocities at the control points so inverting the whole system of Eqs. (47) is only carried out once for each body shape and height. As far as carrying out the integrals in (39) is concerned we truncate them at some point $r_{\infty} \gg 1$ and then, assuming the far-field form from 2.2, carry out the integrals beyond this point analytically while numerically determining them for $1<r<r_{\infty}$. The value of $r_{\infty}$ used was $r_{\infty}=100$ and this was varied to ensure that there was no sensitivity in the solutions to our choice.

The typical number $N$ of Tchebyshev polynomials required varies with $h$ but is generally between 20 and 24 to give $a_{N} \approx \sqrt{E}$, where $E$ is the least squares error. The expected exponential decay of the coefficients $a_{i}$ can be seen clearly as in Fig. 3. The method was also applied without setting $a_{0}$, $a_{1}$ (i.e. minimising over all $N+1$ Tchebyshev coefficients) to check the far-field and near disc conditions were satisfied even when they were not initially forced. Although the minimisation took longer to converge, the results were identical.

The results are shown in Fig. 4 for a disc with no body shape beneath it and with no pressure jump $(\alpha=0)$. It can be seen clearly that as $h$ decreases the deflection of the layer is increased. The far-field response can also be seen to emerge as $r$ increases. As $h$ is reduced further the layer shape, rather than monotonically tending towards the $30^{\circ}$ far field angle, overshoots it and tends to the far field form from above. This arises from the layer shape having infinite curvature at the disc rim as is shown in the next section. Again it should be noted here that for the small $h$ values in Fig. 4 the assumption of an inviscid core is no longer physical.

Also shown, in Fig. 5, is an example of the disc with a body shape beneath it. The shape used was that of a sphere joined to the disc by a short stem. The results show that the presence of a small sphere makes a limited difference to the layer shape, particularly for anything but small $h$. For a large sphere, the layer shape is deflected up further than usual, effectively bringing the ground nearer.

The physically more relevant case is the one with a pressure jump and results are presented in Figs. 6-9 for a variety of heights and values of $\alpha$. Several aspects, when compared to the $\alpha=0$ results are apparent. The far field 


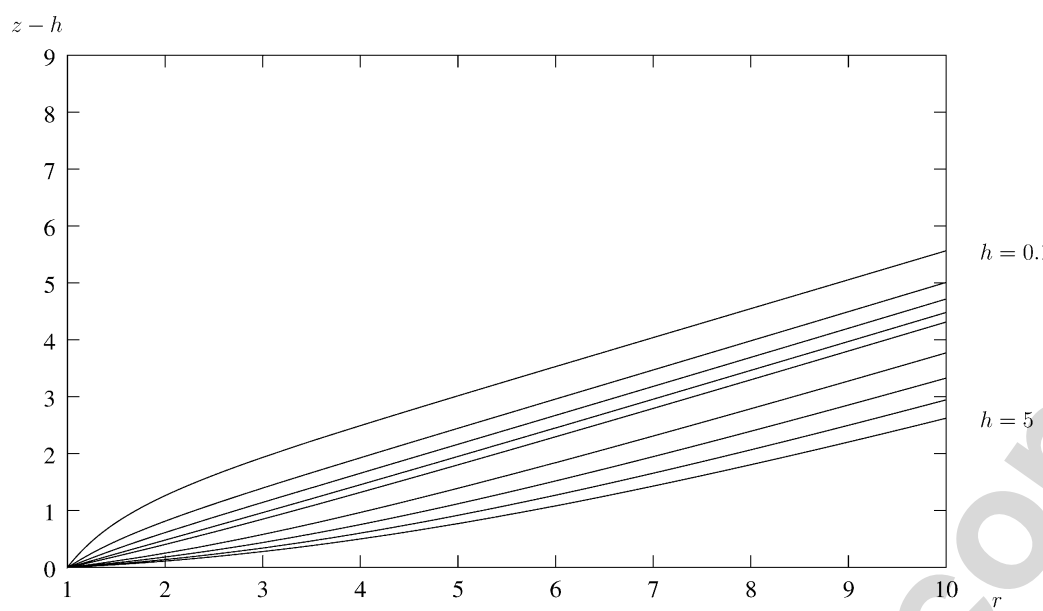

Fig. 4. Layer shapes beyond a rotating disc at various distances $h$ from the ground. $h$ takes the values (from bottom to top) 5, 4, 3, 2.5, 2, 1.5, 1, $0.75,0.1$.

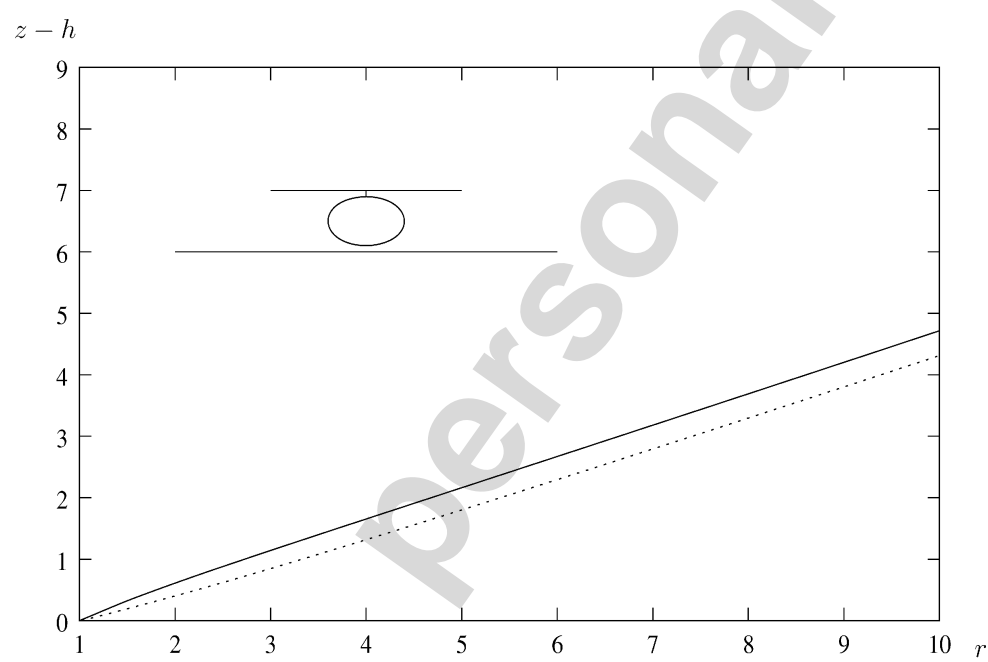

Fig. 5. Comparison of the layer shapes for a disc at a height $h=1$ with and without a spherical body shape. The body shape is shown and has a radius of 0.4 . The solid line is the layer shape with the body present.

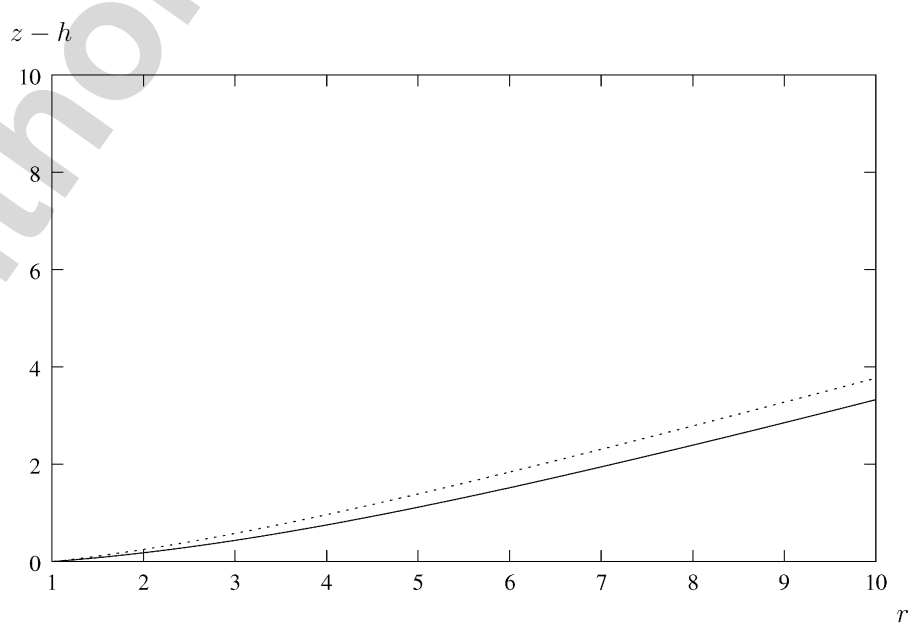

Fig. 6. Comparison between the layer shapes for the pressure jump case (solid line) and the no pressure jump case (dotted line) for $h=2$ with $\alpha=1$. 


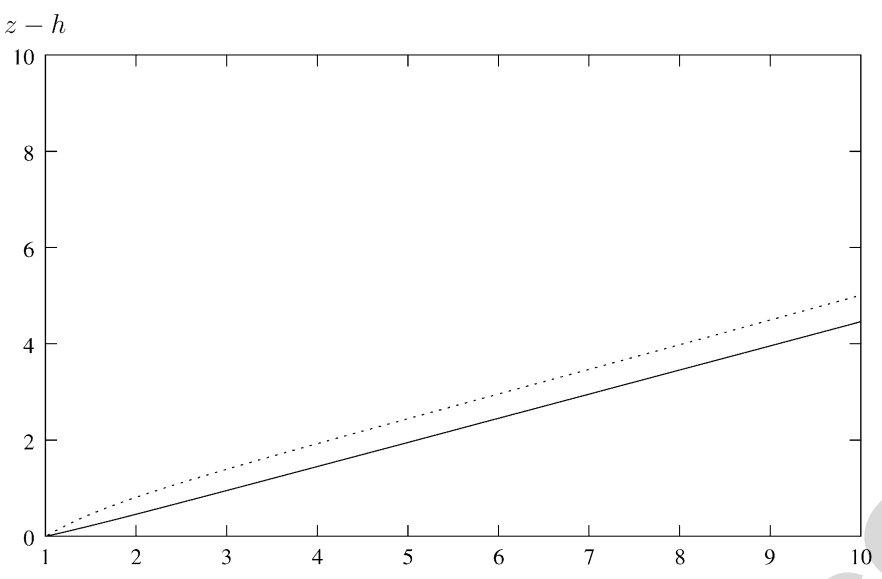

Fig. 7. Comparison between the layer shapes for the pressure jump case (solid line) and the no pressure jump case (dotted line) for $h=0.3$ with $\alpha=1$.

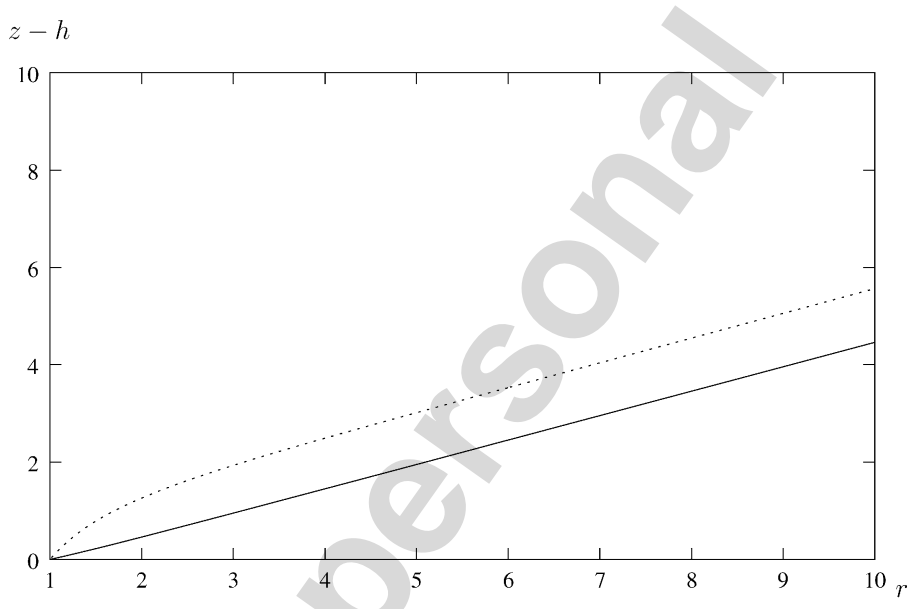

Fig. 8. Comparison between the layer shapes for the pressure jump case (solid line) and the no pressure jump case (dotted line) for $h=0.1$ with $\alpha=1$. Note the similarity in the linear layer shape for the pressure jump case here and for $h=0.3$ case in Fig. 7 .

result is seen to be still holding for large $r$, giving the slope $\pi / 6$ of Section 2.2. The same trends again appear with the deflection of the layer shape increasing as $h$ decreases. The new results generally lie beneath the original ones, and the larger the designated value of $\alpha$ the less the overall deflection. For small $h$ values we no longer have the abrupt change in the layer shape at the disc rim and we also no longer see the overshooting of the far field solution. It seems clear that for large $\alpha$ the right-hand side of (10) dominates, requiring $Z^{\prime \prime}(r)$ to be zero to leading order and hence $Z(r)$ to be linear in $r$. The tying together of the two main slopes, zero at the disc and $\pi / 6$ in the far-field, should thus be of future interest, given the near-linear results for the wake shape in the computations.

\subsection{Small $h$}

As can be seen from the numerical results the deflection of the layer shape becomes quite severe near the disc rim for $h \ll 1$. To examine why the flow solution behaves in this manner and as a check on the numerical solution we now turn to the limit of small $h$. We consider an $\mathrm{O}(h)$ by $\mathrm{O}(h)$ region around the disc rim by writing $z=h+h \hat{z}$, $r=1+h \hat{r}, Z(r)=h Z_{0}(\hat{r})+h^{2} Z_{1}(\hat{r})+\cdots$ and, in view of the infinite disc results from 2.3, expand the velocities and pressure as

$$
\begin{aligned}
& \left(u^{+}, v^{+}, p^{+}\right)=\left(u_{0}^{+}+\cdots, v_{0}^{+}+\cdots, p_{0}^{+}+\cdots\right), \\
& \left(u^{-}, v^{-}, p^{-}\right)=\left(\frac{u_{-1}^{-}}{h}+u_{0}^{-}+\cdots, v_{0}^{-}+\cdots, \frac{p_{-2}^{-}}{h^{2}}+\frac{p_{-1}^{-}}{h}+\cdots\right)
\end{aligned}
$$




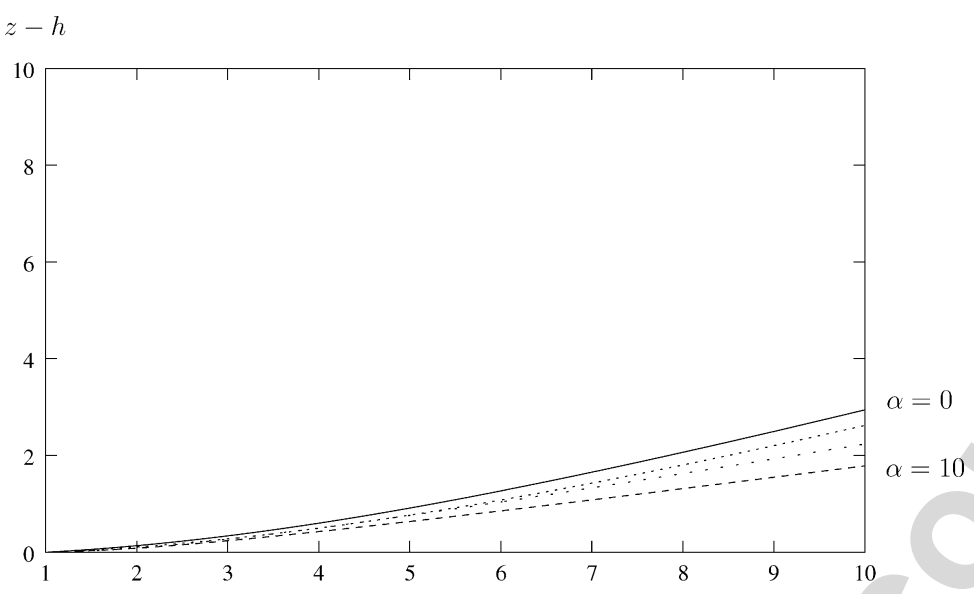

Fig. 9. Comparison between the layer shapes for $h=5$ and varying the value of $A$ from $\alpha=0$, the uppermost solid line, through $\alpha=0.1$ and $\alpha=1$ to $\alpha=10$ the lowest line.

above and beneath the disc and layer respectively. We then need to solve the Euler equations subject to

$$
\begin{aligned}
& v^{-}=0 \quad \text { when } \hat{z}=-1, \\
& v^{-}=c \quad \text { when } \hat{z}=0, \hat{r}<0, \\
& v^{-}-Z_{1}^{\prime}(\hat{r}) u^{-}=c \quad \text { when } \hat{z}=0, \hat{r}>0, \\
& p^{+}=p^{-} \quad \text { on } \hat{z}=Z(r), \\
& \left(u_{-}, v_{-}, p_{-}\right) \rightarrow \text { infinite disc solution } \quad \text { as } \hat{r} \rightarrow-\infty,
\end{aligned}
$$

which are the no penetration condition on the ground, constant entrainment into the disc boundary layer, the known normal entrainment into the layer, pressure continuity across the layer. The final condition (55) actually emerges as part of the solution. Substituting the velocity expansions into the Euler equations and applying the relevant conditions yields the leading order terms beneath the system immediately as, $u_{-1}^{-}=-c / 2, p_{-2}^{-}=-c^{2} / 8, Z_{0}(\hat{r})=0$. In order to maintain pressure continuity it is necessary to add an $\mathrm{O}\left(1 / h^{2}\right)$ constant to $p^{+}$to match with this region.

The next order equations yield Laplace's equation for $v_{0}^{-}$and $p_{-1}^{-}$subject to

$$
\begin{aligned}
& v_{0}^{-}=0 \quad \text { on } \hat{z}=-1, \\
& v_{0}^{-}=c \quad \text { on } \hat{z}=0, \hat{r}<0, \\
& v_{0}^{-}=c\left(1-\frac{Z_{1}^{\prime}}{2}\right) \quad \text { on } \hat{z}=0, \hat{r}>0, \\
& p_{-1}^{-}=0 \quad \text { on } \hat{z}=0, \hat{r}>0,
\end{aligned}
$$

where the fourth of these conditions is necessary as $p_{-1}^{-}$is not a constant and is too large to be matched by the flow solution above the layer.

To solve this problem we map the region into the complex $\bar{z}$-plane using the mapping $\bar{z}=\mathrm{e}^{\pi \tilde{z}+\mathrm{i} \pi}$, where $\tilde{z}=\hat{r}+\mathrm{i} \hat{z}$. This leaves us with a half-plane problem subject to mixed boundary conditions along the $\bar{x}$-axis. So we need to find $v_{0}^{-}$and $p_{-1}^{-}$, both satisfying the Cauchy-Riemann equations, and subject to

$$
\begin{aligned}
& v_{0}^{-}=0 \quad \text { on } \bar{y}=0, \bar{x}>0, \\
& v_{0}^{-}=c \quad \text { on } \bar{y}=0,-1<\bar{x}<0, \\
& p_{-1}^{-}=0 \quad \text { on } \bar{y}=0, \bar{x}<-1 .
\end{aligned}
$$

Now consider the complex function

$$
g(\bar{z})=(\bar{z}+1)^{-1 / 2}\left(p_{-1}^{-}+\mathrm{i} v_{0}^{-}\right),
$$


analytic in the upper-half plane $\bar{y}>0$. Considering (63) for $\bar{x}<1$ and $\bar{x}>1$, using Cauchy's integral formula to relate real and imaginary parts of $g(\bar{z})$ and applying conditions (60)-(62), we obtain the relations,

$$
\left.\begin{array}{ll}
|\bar{x}+1|^{-1 / 2} v_{0}^{-} & (\bar{x}<-1) \\
|\bar{x}+1|^{-1 / 2} p_{-1}^{-} & (\bar{x}>-1)
\end{array}\right\}=\frac{c}{\pi} \int_{-1}^{0} \frac{\mathrm{d} \xi}{(\xi+1)^{1 / 2}(\xi-\bar{x})} .
$$

Carrying out this integral, using the substitution $\xi=\theta^{2}-1$, and mapping back to the original plane gives the velocity on $\hat{z}=0, \hat{r}>0$ as

$$
v_{0}^{-}(\hat{r}>0,0)=\frac{2 c}{\pi} \arctan \left(\frac{1}{\sqrt{\mathrm{e}^{\pi \hat{r}}-1}}\right),
$$

and the layer shape, from (58), as

$$
Z_{1}(\hat{r})=2 \hat{r}-\frac{4}{\pi} \int_{0}^{\hat{r}} \arctan \left(\frac{1}{\sqrt{\mathrm{e}^{\pi \xi}-1}}\right) \mathrm{d} \xi .
$$

So right at the disc rim, i.e. for $\hat{r} \ll 1$,

$$
Z_{1}(\hat{r})=\frac{8}{3 \pi^{1 / 2}} \hat{r}^{3 / 2}+\cdots
$$

This has infinite curvature at the rim and explains the rapid change in the layer shape in the numerical results near the disc. The result also indicates why the mapping $x=-1 / r^{2}$ in the parameterisation of the layer shape works better for small $h$ than the $-1 / r$ one. The other limit of interest is as $\hat{r} \rightarrow \infty$. There $v_{0}^{-} \rightarrow 0$ and the layer shape is given by

$$
Z_{1}(\hat{r})=2 \hat{r}+\cdots
$$

So we have just outside the disc rim region, i.e. for $\hat{r} \gg 1$,

$$
Z(r)=h+h^{2}\left(\frac{2(r-1)}{h}\right)+\cdots=h(1+2(r-1))+\mathrm{O}\left(h^{2}\right)
$$

which, when compared to the far field result $Z(r) \sim r / \sqrt{3}$, explains the overshooting of the far field angle seen in the numerical results. A comparison of this small $h$ analysis and the numerical calculation is shown in Fig. 10 where good agreement can be seen.

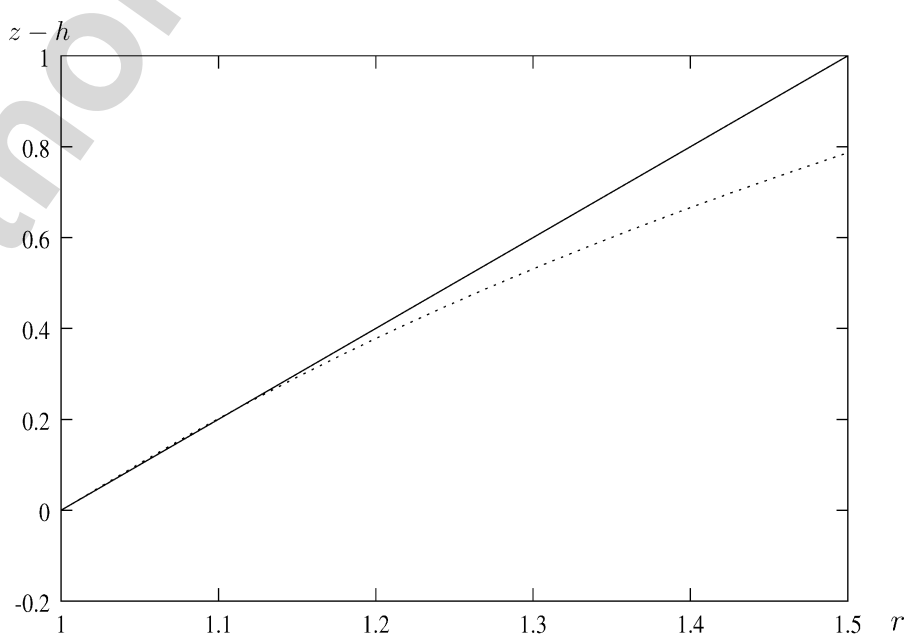

Fig. 10. Comparison between the small $h$ analytical result near the disc rim (solid line) and the layer shape for $h=0.1$ from the numerical calculations. 


\section{Further comments}

The flow produced by an axisymmetric disc, with or without a modelled fuselage (underbody), rotating above a horizontal ground has been considered, specifically concentrating on the distortion of the free layer shape beyond the disc rim induced by the presence of the ground. The limiting cases of large radial distances, a nominally infinite disc and small ground clearances have been examined, while an integral formulation of the full inviscid problem has been applied which includes or excludes a pressure jump across the free layer. The formulation was solved numerically in all cases, considering several magnitudes of the pressure jump as well as various values of the relative ground clearance and the influence of an underbody. The main findings are the $30^{\circ}$ inclination of the straight free-layer shape from the horizontal in the far-field, the quite rapid approach radially to that inclination angle and the increasing influence found from the presence of the underbody as the ground is approached, at least in the settings examined here.

The current approach ignores viscosity and compressibility, both of which may be important in reality. It also ignores the possibility of inner-outer interaction, whereby the viscous layer interacts directly with the inviscid solution rather than passively as in the current case. Inner-outer interaction yields a configuration similar to that considered by Smith and Timoshin [24], Purvis and Smith [25] and Purvis [26] in related two-dimensional contexts but would require computing the viscous layer coupled with the inviscid solution, with the entrainment into the layer being dependent on the outer inviscid solution which in turn depends upon the entrainment. This certainly becomes important in the case when the disc is replaced by a system of rotor blades as, amongst other things, the outer solution determines where the vortices shed from the trailing edge of one blade impinge upon the leading edge of the next and therefore can have a great influence on the solution as a whole. Another important effect is that of turbulent layers, a study of which is undertaken by McDarby [27].

\section{Acknowledgements}

Thanks are due to Susan Brown, Robert Bowles, John Elliott, Anatoly Ruban, Spencer Sherwin, Sergei Timoshin and the referees for their interest and comments.

\section{References}

[1] A.R.S. Bramwell, Helicopter Dynamics, Edward Arnold, 1976.

[2] J. Seddon, Basic Helicopter Dynamics, BSP Professional Books, Oxford, 1990.

[3] S. Newman, The Foundations of Helicopter Flight, Edward Arnold, 1994.

[4] A.T. Conlisk, Modern helicopter aerodynamics, Annu. Rev. Fluid Mech. 21 (1997) 515-567.

[5] J. Zbrozek, Ground effect on the lifting rotor, Aero. Res. Counc. Rep. and Memor, no. 2347c.

[6] S.E. Widnall, T.M. Barrows, An analytical solution for two and three dimensional wings in ground effect, J. Fluid Mech. 41 (1970) $769-792$.

[7] M.J. Lighthill, A simple fluid flow model of ground effect on hovering, J. Fluid Mech. 93 (1979) $781-797$.

[8] J.N. Newman, Analysis of small-aspect-ration lifting surfaces in ground effect, J. Fluid Mech. 117 (1982) 305-314.

[9] E.O. Tuck, T.M. Bentwich, Sliding sheets: lubrication with comparable viscous and inertia forces, J. Fluid Mech. 41 (1983) 769-792.

[10] M.A. Jones, Mechanisms in wing-in ground effect aerodynamics, Ph.D. Thesis, University of London, 2000.

[11] M.A. Jones, F.T. Smith, Fluid motion for car undertrays in ground effect, J. Engrg. Math. 45 (2002) 309-334.

[12] F.T. Smith, S.N. Timoshin, Blade-wake interactions and rotary boundary layers, Proc. Roy. Soc. London Ser. A 452 (1996) $1301-1329$.

[13] H. Glauert, The Elements of Aerofoil and Airscrew Theory, Cambridge University Press, 1937.

[14] J.M. Owen, R.H. Rogers, Flow and Heat Transfer in Rotating-Disc Systems, vol. 1: Rotor-Stator Systems, John Wiley, 1989.

[15] F.T. Smith, A note on a wall jet negotiating a trailing edge, Quart. J. Mech. Appl. Math. XXXI (4) (1978) 474-479.

[16] D. Papageorgiou, F.T. Smith, Non-linear instability of the wake behind a flat plate placed parallel to a uniform stream, Proc. Roy Soc. London Ser. A 419 (1988) 1-28.

[17] R. Debuchy, A. Dyment, H. Muhe, P. Michau, Radial inflow between a rotating and a stationary disc, Eur. J. Mech. B Fluids 17 (1998) 791-810.

[18] P.M. Morse, H. Feshbach, Methods of Theoretical Physics, vols. I and II, McGraw-Hill Book Company, 1953.

[19] I.S. Gradstein, I.M. Ryzhik, Tables of Integrals, Series and Products, Academic Press, 1965.

[20] H. Lamb, Hydrodynamics, Cambridge University Press, 1932.

[21] J.L. Hess, A.M.O. Smith, Calculation of potential flow about arbitrary bodies, Prog. Aero. Sci. 8 (1967) 1-138.

[22] J.L. Hess, Panel methods in computational fluid dynamics, Annu. Rev. Fluid Mech. 22 (1990) 255-274.

[23] B.D. Bernardinis, D.W. Moore, A ring vortex representation of an axi-symmetric vortex sheet, in: M.Y Hussain, M.D Salas (Eds.) Studies of Vortex Dominated Flows, 1986, pp. 33-43.

[24] F.T. Smith, S.N. Timoshin, Planar flows past thin multi-blade configurations, J. Fluid Mech. 324 (1996) 355-377. 
[25] R. Purvis, F.T. Smith, Planar flow past many blades in ground effect, Quart. J. Mech. Appl. Math. 57 (1) (2004) 137-160.

[26] R. Purvis, Rotor blades and ground effect, Ph.D. Thesis, University of London, 2002.

[27] J. McDarby, The turbulent boundary layer on a set of rotor blades in an enclosed space, Ph.D.Thesis, University of London, 2004. 